\title{
Microscopic Simulation-Based High Occupancy Vehicle Lane Safety and Operation Assessment: A Case Study
}

\author{
Chao Li (D), Mohammad Karimi, and Ciprian Alecsandru (iD \\ Department of Building, Civil and Environmental Engineering, Concordia University, Montréal, QC, Canada H3G 2 W1 \\ Correspondence should be addressed to Chao Li; chaoli0351@gmail.com
}

Received 2 June 2017; Revised 18 January 2018; Accepted 4 March 2018; Published 5 April 2018

Academic Editor: Alain Lambert

Copyright (C) 2018 Chao Li et al. This is an open access article distributed under the Creative Commons Attribution License, which permits unrestricted use, distribution, and reproduction in any medium, provided the original work is properly cited.

\begin{abstract}
This study proposes two general alternative designs to enhance the operation and safety of High Occupancy Vehicle (HOV) lanes at junctions with bus terminals or parking lots. A series of analysis tools, including microscopic simulation, video-based vehicle tracking technique, and Surrogate Safety Assessment Model (SSAM), are applied to model and test the safety and operational efficiency of an HOV road segment near a bus terminal in Québec as a case study. A metaheuristic optimization algorithm (i.e., Whale Optimization Algorithm) is employed to calibrate the microscopic model while deviation from the observed headway distribution is considered as a cost function. The results indicate that this type of HOV configurations exhibits significant safety problems (high number of crossing conflicts) and operational issues (high value of total delay) due to the terminal-bound buses that frequently need to travel across the main road. It is shown that the proposed alternative geometry design efficiently ameliorates the traffic conflicts issues. In addition, the alternative control design scheme significantly reduces the public transit delay. It is expected that this methodology can be applied to other reserved lane configurations similar to the investigated case study.
\end{abstract}

\section{Introduction}

The HOV lane represents a restricted usage traffic lane reserved for vehicles carrying a predetermined number of occupants. The implementation of an HOV lane system targets mobility improvement of both current and future roadway networks. Considering over forty years of deployment of HOV lanes, it has been proven that reserved lanes contribute to mitigating traffic congestion in urban areas and reduce the person-hour delay effectively $[1,2]$. However, many problems related to various implementations of HOV lanes have been identified. These problems can be roughly classified into two categories, the reduction of capacity (for the non-HOV users) and potential traffic safety issues, respectively. The former category may include increased congestion on the adjacent General Purpose (GP) lanes and/or reduction of vehicle speeds due to the merging maneuvers of High Occupancy Vehicles into the GP lanes. The latter category is mainly related to the lane changes at prohibited locations, especially in the proximity of junctions with other road facilities, such as bus terminals or parking lots [3].
Currently, efforts are continually being made to explore the new ways to improve the operation and safety of $\mathrm{HOV}$ facilities. However, there is no universally accepted method to evaluate the effectiveness of safety of certain HOV facilities [4]. Some studies focused on the HOV safety evaluation based on the statistical analysis of accidents data during long periods [5]. Several studies examined the safety of HOV facilities with respect to different types of geometric design based on the collision and driving behavior (i.e., lane-changing) data [6, 7]. Nevertheless, obtaining reliable accident data over a long enough period is not always possible, especially for recently deployed facilities. A reliable accident-based analysis takes a long time to establish and thus is not suitable for current urban traffic system development. In addition, many characteristics of the urban traffic system may change over time (e.g., traffic demand volumes, road alignments, traffic mix, etc.), and this might require an expedited method to assess the existing traffic conditions. Accordingly, using conflict analysis as a method of safety assessment is preferable, as it makes analyzing the safety 
improvement before implementing any treatment in the real world possible.

However, the geometric configuration of an HOV facility has significant impacts on the safety performance [7]. For instance, conducting the before-after study of converting the continuous access to limited access of lane changes in $\mathrm{HOV}$ lanes has shown a significant decrease in conflict occurrence. Therefore, the HOV facilities with limited access are expected to be safer than those with continuous access. To validate this conclusion, more studies must be conducted. However, there is limited opportunity for researchers to conduct beforeafter studies of road facilities with respect to the geometric modification, because they are too infrequent. Therefore, utilizing simulation tools may be an effective remedial measure to overcome the limitation of data availability and to evaluate the impacts of potential geometric alignment changes of existing facilities. Several studies have introduced the evaluation of safety or capacity of HOV facilities utilizing microsimulation [3, 8, 9]. However, these studies mainly focused on the analysis results of the study areas. Therefore, it is necessary to develop a systematic assessment method for HOV lanes. In particular, the HOV deployment on arterials in the proximity of the terminals and parking lots can be conducted using real-word data to calibrate a microscopic simulation model.

In this study, a VISSIM microsimulation model is developed to test the safety and operational efficiency of an urban HOV facility near a bus terminal in Québec, Canada. This model is calibrated by employing a metaheuristic optimization algorithm-Whale Optimization Algorithm (WOA)-to minimize the deviation of simulations results from the observed data. Two general alternative network designs are proposed for comparison analysis (i.e., one modifies the existing road geometric alignment; another one proposes a change in the existing traffic control strategy). To assess the road safety impact of the proposed alternative designs, the Surrogate Safety Assessment Model (SSAM) is applied to compare the simulated vehicle conflicts between the existing network and the alternative solutions. The results indicate that the status quo of the study area exhibits a safety problem due to high interactions between buses and passenger cars. The proposed alternative geometry design efficiently eliminates the traffic conflict. In addition, the alternative control design scheme significantly reduces the public transit delay.

\section{Literature Review}

Traditionally, most traffic safety studies employed statistical analysis of accident records within a given study area [10-13]. Several studies pointed out the drawbacks of using authority reported crash data for safety evaluation, for example, the lack of ability to evaluate the safety of traffic facilities yet to be built or to assess the traffic remediation solution yet to be applied in the field. In addition, the seldom and random occurrence of traffic accidents lead to the slowness of establishing analysis [14] or the lack of ability to deduce the crash process $[15,16]$. On account of these drawbacks, an alternative safety evaluation approach which includes the computer microsimulation modeling of vehicle interactions was developed over the past couple of decades. This approach was possible mainly due to the advancements in computing technologies that allowed the development of enhanced traffic simulation models to be able to replicate vehicle interactions through modeling complex driving behaviors [16, 17]. A significant advantage of simulation-based safety analysis is that microsimulation models can easily generate and measure various safety performance indicators [18, 19]. The typical safety performance indicator is the vehicular conflict, given that conflicts can be observed more frequently than crashes and that their frequency is expected to be correlated with the crash occurrence [14, 20-22]. Various studies have validated the statistical significance and correlation between conflicts and accidents [23-26].

A dedicated tool, namely, SSAM was developed by Federal Highway Administration (FHWA) to automatically identify, classify, and evaluate the severity of the simulated traffic conflicts [14]. Several studies showed that by combining VISSIM and SSAM a reliable tool for traffic safety evaluation can be used, provided that a consistency between the field observed and simulated conflicts is observed [27,28]. Another study proposed a two-step calibration procedure of VISSIM (Wiedemann model) to enhance correlation between simulated and field-measured conflicts [29]. Therefore, if the simulation model is properly calibrated, it can be used to represent reliably the real-world traffic network in terms of both operation and safety parameters.

\section{Methodology}

3.1. Modeling of Geometry and Flow. Typically, more detailed information contained in the simulation model contributes to capturing more reliably the traffic conditions at a given study area. This is especially important for a traffic safety simulation model, which requires good accuracy of both simulated capacity and vehicle performance.

The basic input to this model is represented by the road characteristics (i.e., the number of lanes on each direction, the lane separation type, and the position of access). In this study, the links and connectors of the study area were built in VISSIM by means of an aerial photo from Google Maps ${ }^{\circledR}$. Some details of the geometry, for example, the access position of the public transit terminal, were measured on the field and were compared with the field-recorded videos to ensure the accuracy. Similarly, the position of the reserved lane was collected on the field and included in the simulation model.

Traffic flow is another important input parameter as it relates to the road capacity, one of the potential calibration variables. Traffic flows were measured using the videos recorded at the study area-the following data was collected: the vehicle counts of each lane, vehicle routes within the study area, and the vehicle types (e.g., bus, truck, and passenger cars). In this study, in order to smooth out random variations in flows, while maintaining good precision, the vehicle flows were recorded and input into the model in five-minute increments. An additional five-minute period without vehicle demand was included at the end of each simulation scenario to avoid truncating the analysis period observed in the field. To model the observed vehicle composition, road users were 
identified and classified into three categories, passenger cars, buses, and trucks, respectively. The basic vehicle characteristics, for example, the acceleration rate, vehicle length, and vehicle weight of each vehicle type, can be modeled separately in VISSIM so as to reflect the traffic more realistically. To determine individual vehicle routes, vehicles were tracked in from the videos generated by three cameras that were used to cover the whole study area. The route of each vehicle in the simulation was assigned in strict accordance with the path observed on the video recordings to ensure a realistic representation of the study area.

3.2. Modeling of Traffic Signal. The peak hour traffic signal cycle length and the red, amber, and green time intervals on each direction were collected on the field and modeled in VISSIM. In this study, a fix-cycled signal program was built and set at the intersection to replicate the traffic light at the study area. Some additional signal control strategy was used in this study to improve the network performance; for example, a fix signal cycle contains a protected left-turn phase at the intersection and a pulse-triggered signal at the public transit terminal.

To improve the efficiency of public transit, a pulsetriggered signal control was implemented by adding a detector at the exit of the terminal and signal heads linked with the detector near the terminal. An add-on signals design model, namely, Vehicle Actuated Programming (VAP) was programmed to control this actuated signal. Typically, a signal phase of permanent green on the main street and permanent red on the minor road is toggled when no buses are detected. Meanwhile, when the existing buses are detected by the sensor, the signal is programmed to switch to the complementary phase (i.e., green signal on the minor road and red on the main road), thus protecting the movements of buses crossing through multiple lanes.

3.3. Modeling of Right of Way without Signal Control. In VISSIM priority rules are defined to capture the conflicting traffic flows that are not controlled by signals. In this study, the priority rules were set at the entry and the exit zones of the bus terminal, in order to realistically model the access and egress movements of buses as they were observed in the video recordings. Typically, the buses travel to and from the terminal, yielding to the vehicles traveling along the main arterial, and stop in position near the access or exit until acceptable gaps occur on both directions on the main road. Two thresholds are set for the priority rules to confine the crossing of the yielding vehicles, respectively, the minimum headway and the minimum gap time. A yielding vehicle will stop before the stop line until both predetermined thresholds are achieved. The values of the thresholds are determined by reviewing all the accepted gaps and headways by the crossing buses from the video.

The conflict areas are automatically generated in VISSIM where links or connectors overlap. In this study, the priority rules at the conflict areas were set to capture the vehicles approaching the conflict area from the minor road and yielding those from the main road, as typically observed in the field. The gap time needed for crossing at the conflict area was determined similarly by reviewing the video recordings.

Another important VISSIM calibration parameter is the avoid blocking value, which defines the ratio of vehicles that do not stop in the middle of a junction. This value is defaulted to be $100 \%$ in VISSIM; in other words, all the vehicles will follow the rule, not to block the junctions, if there is stopping traffic ahead. However, by reviewing the video recorded at the study area, no vehicle obeyed this rule. Therefore, to reflect the real conditions, this value is set to $0 \%$ for all the conflict areas in simulation models used in this study.

3.4. Modeling of Driving Behavior. Properly modeling of the field observed driving behavior is critical for road safety evaluation, since it directly influences the vehicle interactions in a micro level. Microsimulation tool VISSIM adopted Wiedemann car following model as the main portion for modeling the vehicle longitudinal movement and rule-based laws for modeling of vehicle lateral movement and lane change behavior.

In this study, the Wiedemann 74 model is selected to simulate the urban motorized traffic as suggested by the VISSIM user's manual [30]. This model contains three adjustable parameters, respectively, the average standstill distance, the additive part of safety distance, and multiplicative part of safety distance. Average standstill distance defines the average desired distance between two cars. Additive part of safety distance and multiplicative part of safety distance represent the values used for the computation of the desired safety distance. For the initial simulation, the values of these three parameters are usually defined with the default value. However, they must be calibrated later to suite the real driving behaviors of the study site.

The lane change behaviors are defined by a rule-based model in VISSIM. In this model, the critical parameter that decides whether a lane change would be executed is the minimum headway. A vehicle can only change lane when there is a distance gap arrival at the adjacent lane that is bigger than the predetermined minimum headway. Otherwise, it has to either travel continuously or stop and wait until the occurrence of an enough gap for it in order to merge according to its predefined route. In this study, the value of the minimum headway was determined by reviewing the videos.

Another noticeable parameter defined in the lane change model is the advanced merging; this option is selected in this study; thus more vehicles can change lanes earlier when following their routes, as encountered in the videos.

\subsection{Measurement of Vehicle Speed Distribution by Feature-} Based Tracking. Vehicle speed distribution is an important input parameter for safety simulation. While potentially more accurate, individual vehicle speed on multiple lanes is usually difficult to measure on the field simultaneously with radar devices. Therefore, an alternative method was applied in this study to measure the vehicle speed, which is the video-based feature tracking.

An open-sourced software project, namely, Traffic Intelligence, was used to automatically track and measure the speed of the vehicles caught by the video at the study 

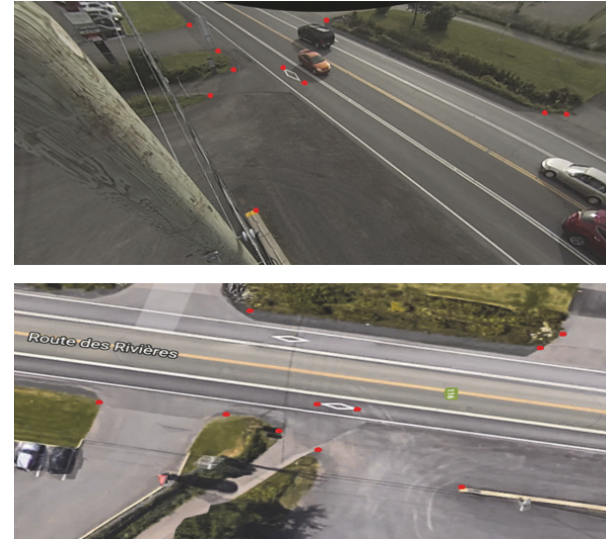

FIgUre 1: Points selected on the video frame to compute homography file.

site [31]. Traffic Intelligence consists of a set of tools that work cooperatively for traffic data processing and analysis, including camera image calibration, feature tracking, and trajectory data analysis.

The feature-based tracking algorithm utilizes a homography file that projects the camera image space to the real-world ground plane. The homography file was created by utilizing a video frame and a corresponding aerial photo with known scale (pixels per meter). In this study, an aerial photo of the study site from Google Maps with known scale of 0.21 pixels per meter was adopted. In total ten noncollinear visible points on the video frame were positioned on the aerial photo; thus, the video image was projected to the aerial photo, and the vehicles tracked in the video were deemed to be tracked in the real-world plane with their speeds. Figure 1 shows the points projected to the aerial photo from the video frame.

Based on the computed homography file, the feature tracking program can be run. The predetermined number of features of each vehicle in the video was detected and tracked frame by frame until the vehicle leaves the video capture area. In order to suppress the interference of the shadows, a mask image was created and toggled with the video image; therefore only the features within the white range of the mask image can be detected, and the shadows can be filtered out. The features that move consistently were then grouped together to generate the trajectory file of each vehicle, and all the trajectories generated from the video were written into a database. The average speed of each vehicle can be easily read by processing their trajectories. Figure 2 shows the feature tracking process by Traffic Intelligence.

3.6. Model Calibration. In order to determine the optimum values for the calibration parameters, an objective function should be defined based on the error between observed data and simulated data. The objective function is the deviation of the simulated gap distribution from the real observed gap distribution. In order to test this goodness of fit (objective), the Chi-square test was employed. In this study, the westbound vehicle gap distribution on the GP lane near the bus terminal was taken as the criterion to calibrate the

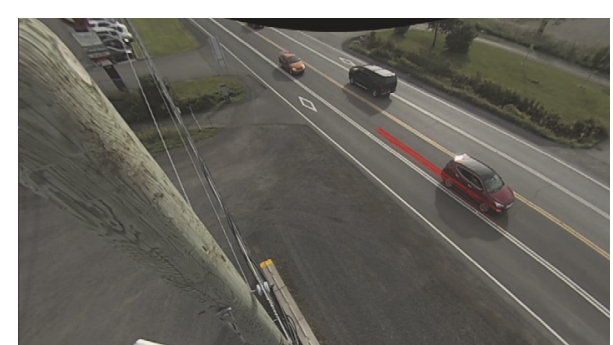

FIGURE 2: Feature tracking process by Traffic Intelligence.

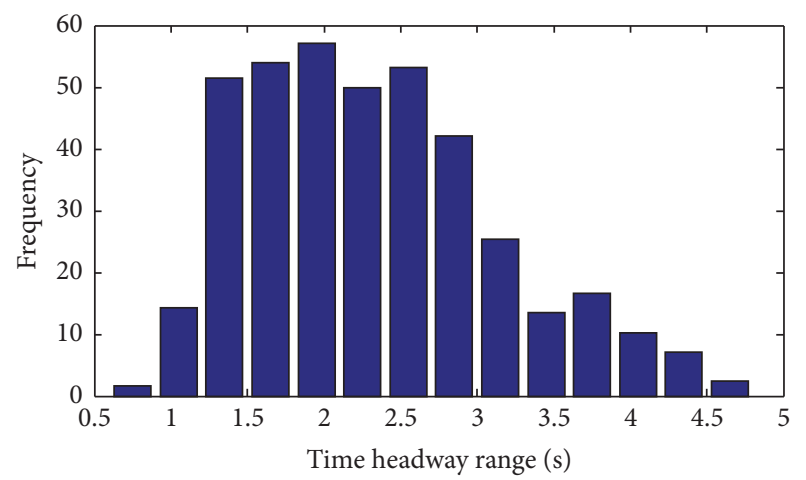

FIgURE 3: Observed vehicle gap distribution.

model, because the vehicle time gap directly reflects the car following behavior. The real vehicle gaps were observed manually from the video using the MPC player that provides milliseconds accuracy. Because the vehicles travel westbound pass through a signalized intersection before they enter the cameras field of view, to eliminate the impact of the red time at the intersection, the time gaps bigger than 5 seconds were ignored. The distribution of all the observed gaps that are smaller or equal to 5 seconds was recorded in a histogram with a sample rate of 0.3 seconds. Figure 3 shows the observed vehicle gap distribution.

In this paper, the Whale Optimization Algorithm (WOA), a metaheuristic nature-based algorithm, is applied to calibrate the model. The deviation of the simulated headway distribution from its' observed distribution is considered as the objective function to be minimized during the calibration process. WOA is inspired by hunting behavior of humpback whales. It is defined as "the simulated hunting behavior with random or the best search agent to chase the prey and the use of a spiral to simulate bubble-net attacking mechanism of humpback whales" [32]. The hunting behavior of whales is representative of the procedure of this algorithm.

The three parameters of the Wiedemann 74 model in VISSIM (i.e., average standstill distance (ASSD), additive part of safety distance (APSD), and multiplicative part of safety distance (MPSD)) which has the highest impact on the model have been selected to be calibrated. Using MATLAB, an optimization toolbox connecting to COM-interface of VISSIM by M-file programming in MATLAB, the calibration process has been accomplished. After 190 simulation runs, the optimal values of parameters were determined to be as 
follows: $\mathrm{ASSD}=1.156, \mathrm{APSD}=0.637$, and $\mathrm{MPSD}=8.079$. For diverse random seeds, the simulation results showed these optimal parameters lead to statistically matching the observed headway distributions with the simulated ones at $90 \%$ confidence level. It is worth mentioning that the simple way to optimize the cost function is exploring the whole possible region of the parameters to find the global minimum, which is extremely time-consuming. For this case study, these optimization parameters took values within the following intervals: ASSD between 0 and 2, APSD between 0 and 1 , and MPSD between 0 and 10. By exploring these intervals, the optimal values were found after nearly 1000 simulation runs.

The lateral movement of buses that merge into the main traffic from HOV lane or travel across the road when an acceptable gap was identified was also calibrated by adjusting the parameters of the priority rule. The minimum gap time and distance headway were set to 6 seconds and 20 meters, respectively, similar to the values observed in the recorded videos. It is noticeable that a part of the terminal-bound buses changed lanes between the reserved HOV lane and the adjacent GP lane before the intersection; this behavior is reflected in the simulation model.

3.7. Simulation Output. VISSIM provides direct output of various kinds of simulation results. In this study, the vehicle delay and trajectory were analyzed to evaluate the operational efficiency and safety of the study area.

Vehicle delay data can be generated by setting vehicle travel time on the defined vehicle routes, which are defined by a Starting Point and an End Point, respectively. For the vehicles that pass through the Starting Point and the End Point, successively, the travel time delays are automatically calculated. The vehicle delays of the interested vehicle routes were then analyzed to evaluate the operational efficiency of the network.

The trajectories of all the simulated vehicles can be generated by VISSIM, and the recorded trajectory data was then analyzed using SSAM, to evaluate the vehicle conflicts within the network. For each simulation run, different simulation random seeds were applied, and the output results were averaged for analysis purposes. This simulation setup scenario accounts for the stochastic properties of the simulation model, thus reflecting real-world traffic behavior more realistically.

3.8. Analyzing Vehicle Conflicts Using SSAM. The vehicle trajectory data collected from VISSIM was used in SSAM to assess the vehicle conflicts detected in the study area. Most studies evaluate traffic safety through two surrogate measures, Time to Collision (TTC) and Postencroachment Time (PET). Values below a commonly accepted threshold of either TTC or PET value indicates a higher probability of collision. SSAM is able to automatically estimate the TTC and PET values of each vehicle interaction and thus to record all potential conflicts. In this study, the TTC and PET were set to 1.5 seconds and 5 seconds, respectively, the values frequently established by previous research studies [20,33].

The detected conflicts were classified into three types, based on the predetermined conflict angles, namely, crossing, lane changing, and rear ending, respectively. The thresholds of the conflict angles were adjusted to 2 degree and 45 degree as suggested by previous studies [8]. Basically, detected conflict which has a conflict angle of 2 degrees or less is defined as rear ending conflict; if the conflict angle is between 2 and 45 degrees, it is detected as lane changing; while if the conflict angle is bigger than 45 degrees, it is recorded as the crossing type. However, due to the peculiarity of geometry of each study area, the link information of all the output conflicts, which was also detected by SSAM, was manually checked to properly determine their type. The three types of conflicts were recorded for subsequent comparative safety analysis.

A built-in filter of SSAM can be applied to screen out the conflicts caused by each measured movement by reading the corresponding link information. The spots where conflicts were detected can be plotted automatically on the toggled network image by positioning the VISSIM network coordinates. The conflicts of different types can be showed in different shapes or colors on the togged map to give a visual estimate of the hotspot areas (i.e., conflicts' frequency and density).

3.9. Summary. The methodology presented in this study introduces a simulation-based approach to evaluate road network safety and efficiency. To apply this methodology, the field traffic conditions are collected, and the detailed information including the field geometry, control strategy, flow, and driving behavior is reviewed. Such basic information is then integrated in a VISSIM simulation model. With an important model parameter, the vehicle speed distributions are obtained using a feature tracking program, namely, Traffic Intelligence. The model is properly calibrated until the output vehicle time gap distribution compared well with the field observed vehicle gap distribution by applying the Chi-square test. The model output vehicle delays are reviewed for network operational efficiency analysis, and the model output vehicle trajectory files are analyzed by SSAM to determine the conflict within the study area thus giving the safety level of the site. Figure 4 shows the flow chart of the methodology used in this study for traffic safety and operational efficiency evaluation.

\section{Case Study}

4.1. Study Area Description. The study area used in this study is a segment of Rte-116, a suburban highway in Lévis, Québec. Evaluations of traffic safety and operations were made at a specific location along the four-lane east-west arterial segment that includes one GP lane and one HOV lane, in both directions. The eastbound reserved lane allows buses and passenger cars with three or more passengers, while the westbound direction has a bus-only lane. The current design of this facility is such that the westbound buses arriving at or departing from the terminal have to travel across the fourlane undivided road. Figure 5 shows the current paths of the buses using the terminal.

The traffic video feeds of vehicles accessing the terminal, the commuter parking lot, and traveling along Rte-116 were collected via GoPro HD video cameras that were installed 


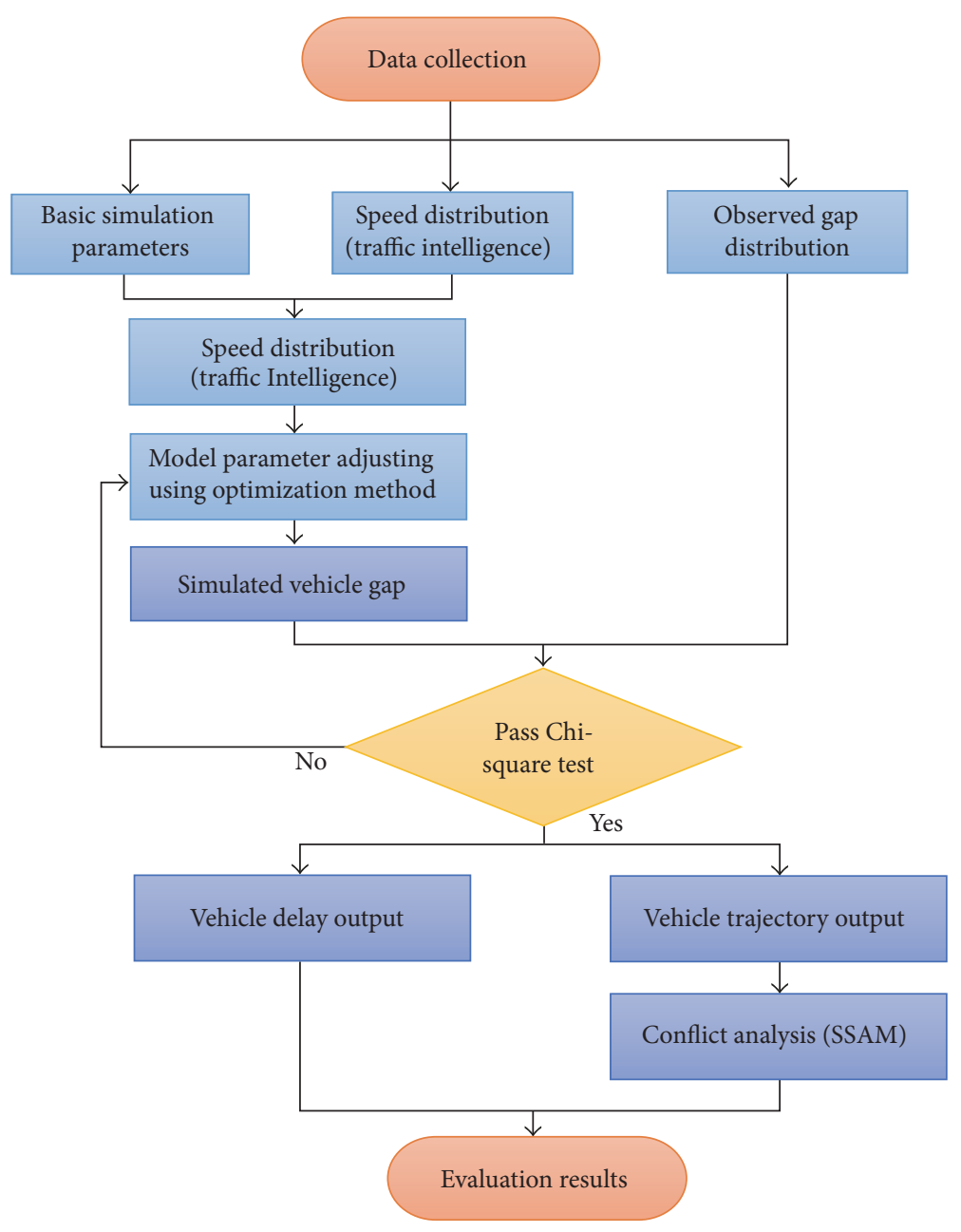

FIGURE 4: Framework of evaluation procedure.

on top of extendable masts along the roadway. Cameras 1 and 2 were both installed at the same location with views opposing each other. The orientations of these two cameras were adjusted to capture east-west traffic that interacts with both access points into and out of the bus terminal. Camera 3 was installed at the proximity of the commuter parking lot entry/exit gate, to capture interactions between main road traffic and vehicles to and from the parking. The positions of the cameras are shown in Figure 5. The video traffic data of the PM peak hour (4:30 pm 5:30 pm) was used in the final analysis of this study.

A probe vehicle was driven several times along the study segments with an arbitrarily selected constant speed. The known speed values were used to calibrate the postprocessing speed detection measuring software, Traffic Intelligence. A fixed 88-second cycle of the traffic signal along Rte-116 at the adjacent intersection (i.e., 40 seconds, red, 40 seconds, green, and 4 seconds, yellow) was measured in the field and used in the simulation model of the study area.

The video files from each camera were processed in 5minute increments to manually determine the distribution of traffic flows during the analysis period. Vehicles were

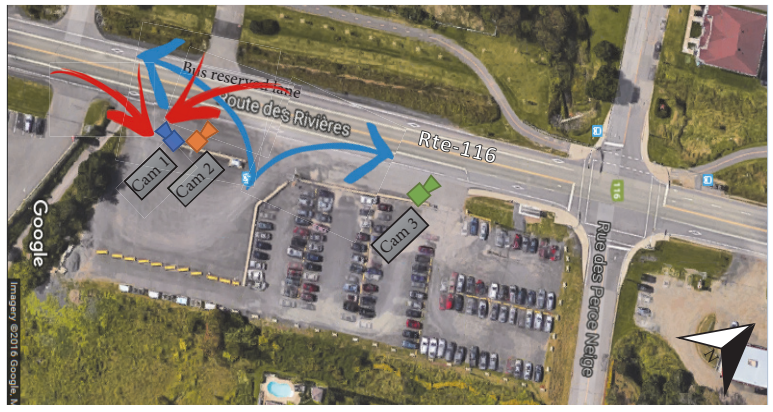

FIGURE 5: Paths of the terminal-bound buses.

distinguished into four types: passenger cars (on the GP lane), buses, trucks, and reserved lane users, respectively. This definition of the traffic mix was necessary to capture more reliably the vehicle interactions in the traffic simulation model (different vehicle types exhibit different driving behaviors in terms of acceleration, minimum headway, etc.). Tables 1 and 2 show a classification of westbound and eastbound 
TABLE 1: Observed traffic flow during the peak hour (4:30 pm 5:30 pm).

\begin{tabular}{|c|c|c|c|c|c|c|c|c|}
\hline \multirow{3}{*}{ Time } & \multicolumn{8}{|c|}{ Average vehicle flows (vehicles/hour) } \\
\hline & \multicolumn{4}{|c|}{ Westbound } & \multicolumn{4}{|c|}{ Eastbound } \\
\hline & Car & Bus & Truck & $\mathrm{HOV}$ & Car & Bus & Truck & $\mathrm{HOV}$ \\
\hline $4: 30 \mathrm{pm} \sim 5: 30 \mathrm{pm}$ & 663 & 16 & 3 & 7 & 338 & 5 & 4 & 36 \\
\hline
\end{tabular}

TABLE 2: Access and egress vehicles during the peak hour (4:30 pm 5:30 pm).

\begin{tabular}{|c|c|c|c|c|c|c|c|c|}
\hline \multirow{4}{*}{ Time } & \multicolumn{8}{|c|}{ Average vehicle flows (vehicles/hour) } \\
\hline & \multicolumn{4}{|c|}{ Westbound } & \multicolumn{4}{|c|}{ Eastbound } \\
\hline & \multicolumn{2}{|c|}{ Bus } & \multicolumn{2}{|c|}{ Parking car } & \multicolumn{2}{|c|}{ Bus } & \multicolumn{2}{|c|}{ Parking car } \\
\hline & Access & Egress & Access & Egress & Access & Egress & Access & Egress \\
\hline $4: 30 \mathrm{pm} \sim 5: 30 \mathrm{pm}$ & 10 & 14 & 0 & 37 & 7 & 3 & 4 & 18 \\
\hline
\end{tabular}

TABLE 3: Vehicles characteristics.

\begin{tabular}{lccccc}
\hline Vehicle type & Length (meter) & Width (meter) & Weight (ton) & Maximum acceleration $\left(\mathrm{m} / \mathrm{s}^{2}\right)$ & Maximum deceleration $\left(\mathrm{m} / \mathrm{s}^{2}\right)$ \\
\hline Car and HOV & $3.75-4.76$ & $1.85-2.07$ & - & 3.5 & -7.5 \\
Bus & 11.54 & 3.17 & $4-12$ & 1.24 & -7.5 \\
Truck & 13.94 & 2.63 & $2.8-40$ & 2.5 & -5.5 \\
\hline
\end{tabular}

traffic flows along the highway, as well as access/egress of the buses using the terminal during the afternoon peak period.

Traffic Intelligence was utilized to measure the vehicle speed. Calibration of the video analysis software was performed using various mask pictures to filter the shadows of the moving vehicles until the measured speeds of the probe vehicle were identical to the observed values. The vehicle speed distributions of both westbound and eastbound vehicles were recorded every five minutes and used as simulation input parameters.

\subsection{Modeling Existing Configuration and Traffic Conditions} (Status Quo). The peak hour traffic was modeled in VISSIM to evaluate traffic safety and operations of the observed arterial segment. Vehicle modals used in the simulation are selected by VISSIM automatically. The vehicle characteristics of the case study are shown in Table 3. To account for the effects of stochastic variation of the model's parameters, ten different simulations with different random seeds were ran, and the average values were used in the analysis.

The average vehicle delay (excluding signal waiting time at intersection) was measured for three types of movements, using the vehicle travel time measurements tool. Movement 1 identifies the westbound traffic on the GP lane. Movement 2 is associated with westbound buses entering the terminal (i.e., buses merging from HOV lane into the GP lane and then crossing the two eastbound lanes). Movement 3 represents westbound buses leaving the terminal (i.e., buses that cross all the four lanes to enter the highway). Vehicle trajectory files were also generated for conflict analysis.

In addition, to evaluate the impact of expected increase in traffic flow on traffic operations (i.e., average vehicle delay) and safety (i.e., conflicts frequency), the same simulation model was used to evaluate similar scenarios, assuming the traffic volume increases in the future by $10 \%, 20 \%$, and $30 \%$

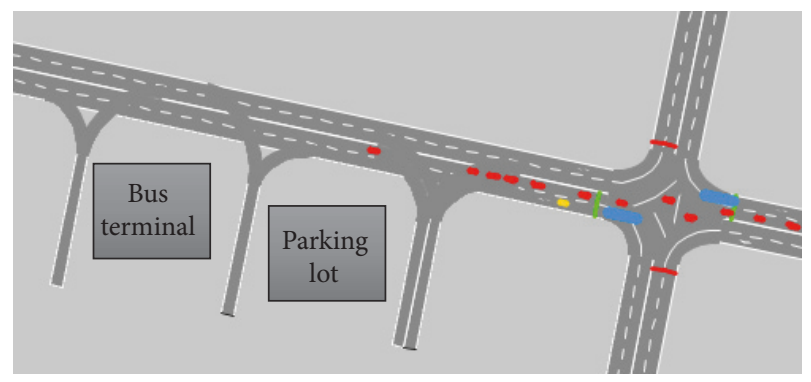

FIGURE 6: The status quo network modeled in VISSIM.

from the current values. Figure 6 represents a snapshot of the VISSIM simulation model using the existing geometric alignment and traffic operations conditions.

4.3. Simulations of Alternative Geometry/Control Designs. The main concern related to traffic safety at the investigated study area pertains to the placement of the reserved lanes on the outside lanes. This configuration leads to multiple lanes crossing when left turns are needed and high occurrence of vehicle interactions was observed especially during congested traffic conditions.

Two alternative designs have been tested to evaluate their potential to mitigate traffic safety and operations issues. Figure 7 shows the VISSIM network layout of the first alternative design. In this model, westbound buses were prohibited to enter or exit the terminal by crossing the highway directly. Instead, an adjacent roadway segment was inserted along the south side of bus terminal, which is directly connected to the minor road. To serve the terminal-bound buses, ten seconds of left-turning signal phase was provided at the intersection on the main road. Similarly, for each 


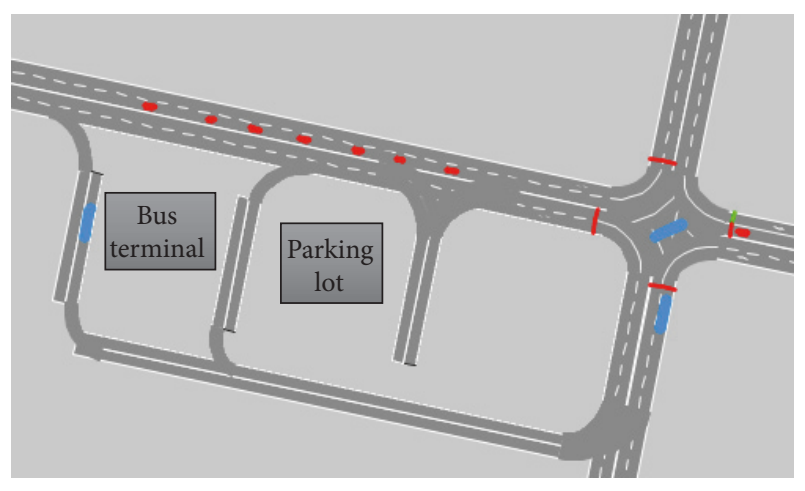

FIGURE 7: VISSIM network of alternative road geometry design.

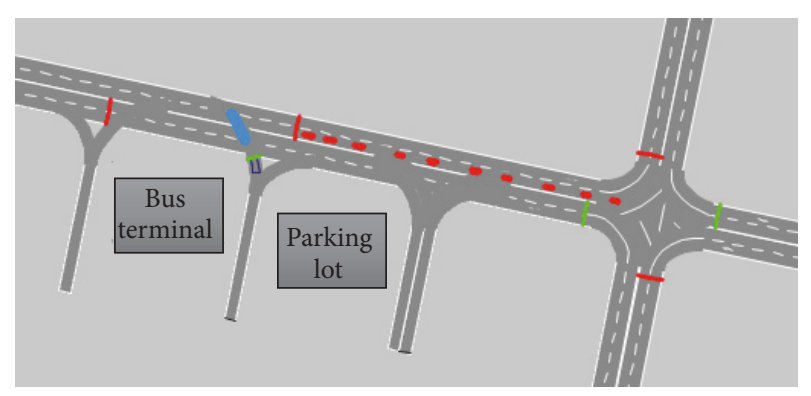

FIGURE 8: VISSIM network of alternative control design.

traffic demand alternative (i.e., current status, $10 \%, 20 \%$, and $30 \%$ increments of vehicular traffic volume), the collected peak hour vehicle flows and speed distributions were used to model the network using ten simulation random seeds. The individual vehicle trajectories and delay measurements of the same movements evaluated for the status quo configuration were collected and used for comparison analysis. Figure 8 shows the VISSIM network layout of the second alternative design. In this model, a loop detector that controls a signal set was added to the existing network. This system was used to control the egress of westbound buses as they leave the terminal. The add-on signal control model VAP was created to program the signal timing. The detector was placed near the exit of the bus terminal. As long as buses are not in the proximity of the sensor, the signal indicates green for the main road to allow east-west traffic and red for the bus exit to prevent the egress buses from traveling across the road directly. When buses are detected at the terminal, exit signal turns green for them and red for traffic on the main road, which allows for protected turns. The red signal on the main road lasts for 10 seconds from the last bus detected and then turns back to green until the next detection. The same vehicle hourly flows previously processed were used in this simulation scenario, and the same ten different simulation random seeds were applied. The delay measurements of the same types of movements and trajectory data were collected for comparative analysis.

4.4. Surrogate Safety Measures of Vehicle Conflicts. SSAM was applied to assess the vehicle conflicts detected in the

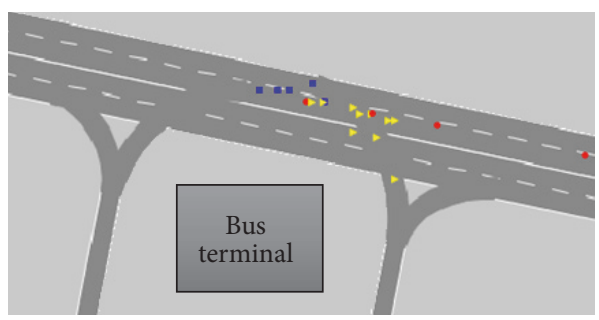

- Rear end conflict
- Lane change conflict
Crossing conflict

FIGURE 9: Conflicts near bus terminal plots on original network.

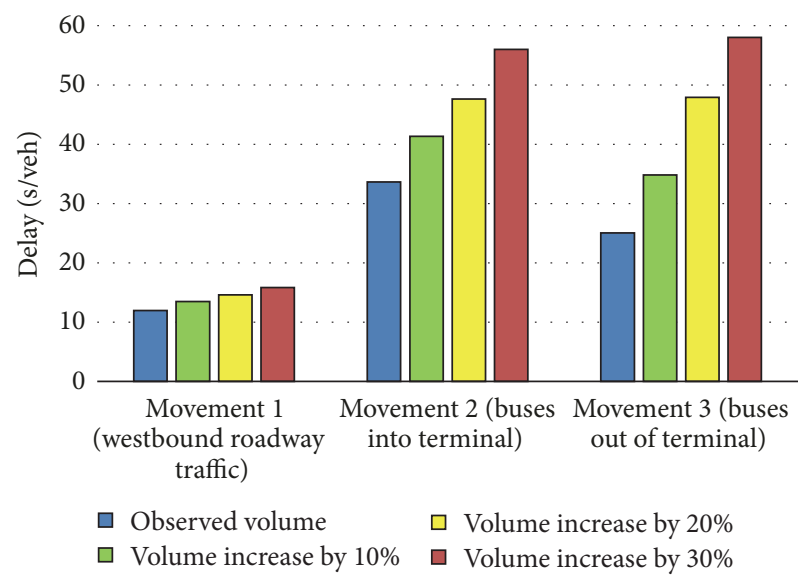

FIGURE 10: Effects of increasing traffic flow on average delay per vehicle.

study area for safety analysis. A built-in filter of SSAM was applied to screen out the conflicts caused by each measured movement by reading the corresponding link information. The spots where conflicts were detected were plotted automatically on the toggled network image by utilizing the VISSIM network coordinates. The conflicts of different types were showed in different shapes on the togged map. Figure 9 shows the spatial distribution of conflicts caused by measured movements near the bus terminal plotted on the original network.

4.5. Comparison Analysis of Safety and Operation. Figures 10 and 11 represent the impact of different traffic volumes on traffic operations (delay) and safety (conflicts).

As intuitively expected, more traffic demand leads to increased average delay. It also shows that of the three types of vehicle interactions analyzed movements labeled 2 and 3 (i.e., associated with buses entering and leaving the terminal) are affected by significantly higher delay than the vehicles moving along the east-west roadway. This is explained by the fact that buses have to make left turns from/into the arterial, and consequently they do not have the default right of way. In addition, traffic safety analysis (i.e., evaluation of vehicular interactions through the SSAM tool) shows that, for all levels of traffic demand, the majority (more than $85 \%$ ) of vehicular 


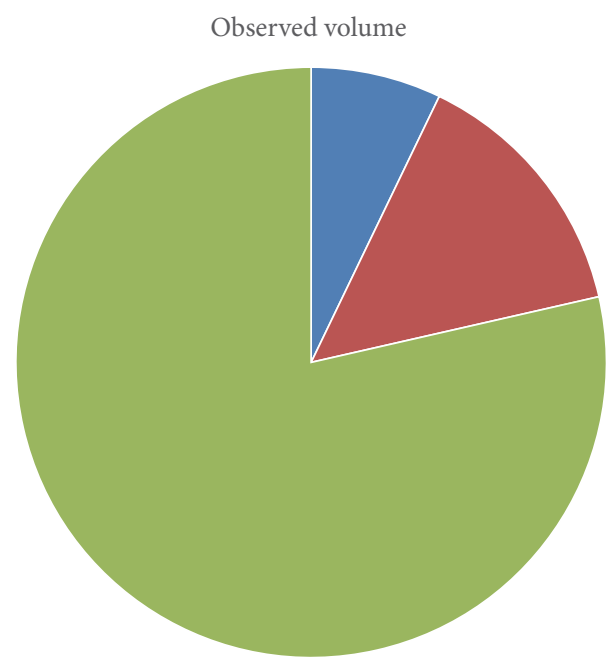

- Movement 1 (westbound roadway traffic)

- Movement 2 (buses into terminal)

- Movement 3 (buses out of terminal)

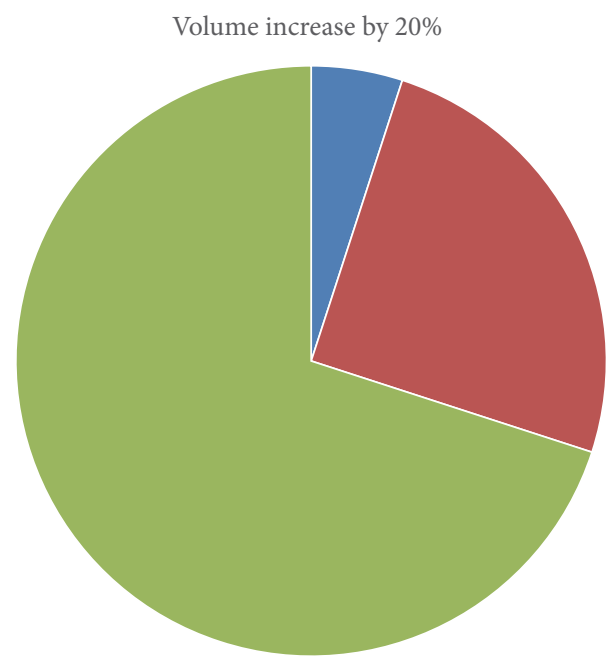

- Movement 1 (westbound roadway traffic)

- Movement 2 (buses into terminal)

- Movement 3 (buses out of terminal)

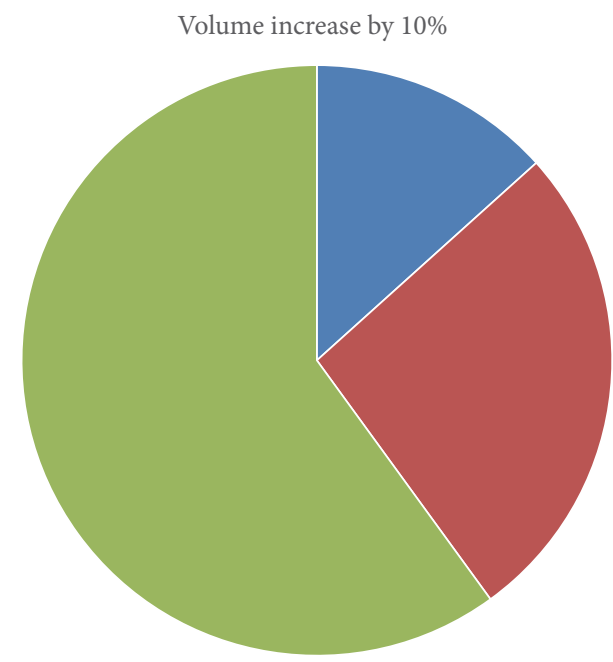

Movement 1 (westbound roadway traffic)

- Movement 2 (buses into terminal)

- Movement 3 (buses out of terminal)

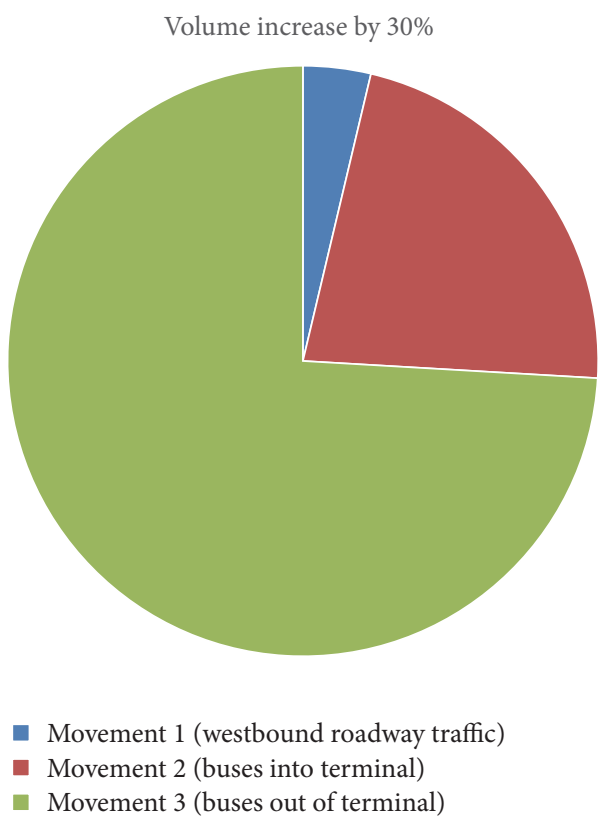

FIGURE 11: Sensitivity analysis of conflicts distribution (current configuration).

conflicts were crossing conflicts associated with the same movements of buses that enter or leave the terminal facility. Moreover, lane-changing conflicts were observed between buses moving from the reserved lane into the GP lane to engage in left-turning maneuvers towards the terminal.

Figure 12 shows the effects of different traffic volumes on traffic operations (magnitude of delay) and safety (frequency of conflicts) when the first alternative scenario was used. As expected, by including a separation median between the two directions of traffic, all vehicular conflicts associated with leftturn movements into and out of the terminal are eliminated.
The sensitivity analysis demonstrates that traffic operations are not impacted by this design. It can be seen that there is a minor positive effect on the average vehicular delay for movement 1 (vehicles traveling westbound on Rte-116), but there is a significant positive effect on the average delay of buses accessing the terminal (i.e., a reduction in delay of about $85 \%$ ). However, this alternative scenario brings a tradeoff for the movements of buses exiting the terminal that are hindered for most traffic flow levels. The additional delay encountered by buses leaving the terminal is due to the fact that, for this design, the westbound egress buses must use 


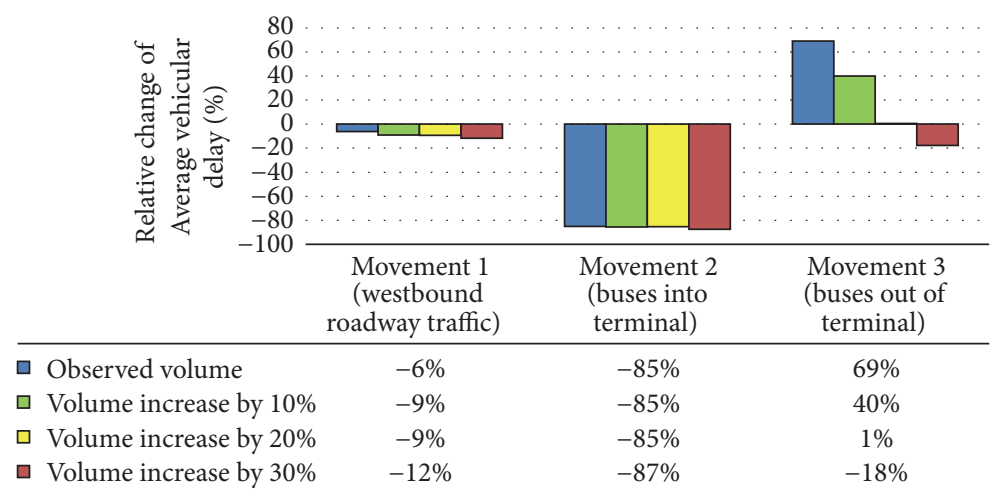

FIGURE 12: Effects of first alternative design on the average delay (separation median).

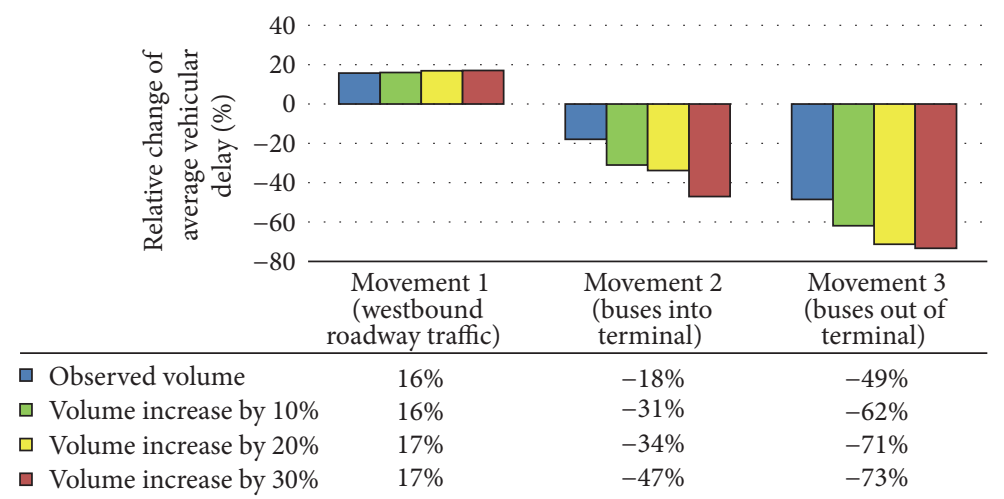

FIGURE 13: Effects of second alternative design on the average delay (traffic control).

the nearby intersection, and the traffic signal timing was not optimized to accommodate left-turning buses from the minor street.

The results for the second alternative design (i.e., controlling the access/egress of buses for Movements 2 and 3 via a bus-triggered traffic control signal, in order to reduce the vehicle interactions with the buses) are shown in Figure 13. It can be seen that this alternative design reduces considerably the delay of buses in and out of the terminal (Movements 2 and 3), while it increases by less than $17 \%$ the delay of vehicles traveling westbound along the arterial (Movement 1).

More importantly, the vehicular conflict analysis of these results shows the elimination of the crossing conflicts (Movements 2 and 3 ) related to buses accessing/leaving the terminal by turning left across the HOV and GP lanes. In addition, this design has no impact on the low conflict occurrence of Movement 1 (vehicles moving westbound on the arterial).

Several aspects of the proposed alternative designs are discussed at the end of this section. The delay of the traffic flow moving westbound on the arterial during the peak period was compared across all three simulation scenarios (i.e., current design, separation barrier, and traffic control alternative). It was found that the traffic control alternative leads to the most negative impact on the vehicular delay. In addition, conflict occurrence between the current design and the proposed traffic control design is not significantly different, due to breaking at red light; it is expected that rear ending conflicts might be more severe. On the other hand, rerouting buses through the intersection via the minor street seems to be the best option, because it eliminates completely all conflicts of left-turning vehicles while its impact on traffic operations might not be significant, since it can be mitigated with optimizing the traffic signal timing plan at the intersection.

To conclude, the existing geometric and traffic signal configurations show that there is a high occurrence of vehicular conflicts for left-turn buses approaching terminal. It can be seen from the results above that, by using the alternative designs, these types of conflicts are eliminated. In addition, the proposed alterations to existing alignment provide benefits for traffic operations because they reduce significantly the average vehicular delay. However, when traffic signals are used to control for protected left-turn buses that are rerouted through the adjacent intersection, an additional analysis of signal delay and optimization is necessary.

Similarly, the analysis of the measured movement 3 (i.e., westbound buses leaving from terminal) identifies a large number of crossing conflicts within the east-west traffic on the main arterial. Elimination of these conflicts can be achieved if this movement is protected either through the traffic signal sensitive to the buses present at the terminal exit, 
or by using the barrier separated geometry that reroutes the buses via the adjacent intersection. The results indicate that the network with alternative control design is the best for departing buses (i.e., the delay is the smallest).

As expected, the sensitivity analysis shows that an increased main arterial traffic volume leads to negative effects on the conflict frequency and average vehicular delay, regardless of the design used, while the alternative designs provide elimination or significant reduction in conflicts.

\section{Concluding Remarks}

This study proposed two general alternative geometry and control designs to improve the operation and safety of High Occupancy Vehicle (HOV) lanes near the bus terminals and parking lots. A VISSIM simulation model was created using the observed field geometry, control strategy, and vehicle flows, and then the vehicle priority rules and driving behaviors were calibrated to reflect correlated parameters observed on the field. An important model parameter, the vehicle speed distribution, was measured by feature-based tracking technique using an open-sourced program, namely, Traffic Intelligence. The model was calibrated using a metaheuristic optimization algorithm (i.e., Whale Optimization Algorithm) with respect to the field-measured vehicles headway distributions. The results showed that this algorithm converged to the optimal parameters faster than searching whole parameters' intervals. The output delay data was used for operational efficiency analysis, and the output trajectory data was analyzed by SSAM to determine the number of vehicle conflicts within the study area. This procedure was applied to test the safety and operational efficiency of an HOV road segment in Lévis, Québec. The peak hour safety and operational traffic conditions of the status quo and of two alternative designs (i.e., geometry and control designs) were analyzed. The results indicate that the existing network configuration exhibits significant safety issues due to the crossing conflicts along the path of buses approaching the terminal across the four-lane arterial road. It was shown that one of the investigated alternative designs may enable the terminal-bound buses to travel on a different path to efficiently eliminate critical vehicular conflict. In addition, it was shown that the alternative control design can be used to reduce the bus delay by giving priority to public transit. It is expected that this methodology can be successfully applied to other similar reserved lanes facilities in the vicinity of the bus stations and parking lots.

\section{Disclosure}

All the viewpoints and errors expressed in this research are solely those of the authors.

\section{Conflicts of Interest}

The authors declare that there are no conflicts of interest regarding the publication of this paper.

\section{Acknowledgments}

This study was funded by Ministère des Transports du Québec (MTQ) through research Project R706.1. The authors would like to thank Matin Giahi Foomani and Gia Hung Lieu from Concordia University for the help in data collection.

\section{References}

[1] C. Fuhs and J. Obenberger, "Development of high-occupancy vehicle facilities: Review of national trends," Transportation Research Record, no. 1781, pp. 1-9, 2002.

[2] M. Menendez and C. F. Daganzo, "Effects of HOV lanes on freeway bottlenecks," Transportation Research Part B: Methodological, vol. 41, no. 8, pp. 809-822, 2007.

[3] A. Guin, M. Hunter, and R. Guensler, "Analysis of reduction in effective capacities of high-occupancy vehicle lanes related to traffic behavior," Transportation Research Record, no. 2065, pp. 47-53, 2008.

[4] J. I. Bauer, C. A. McKellar, J. M. Bunker, and J. Wikman, "High occupancy vehicle lanes-an overall evaluation including Brisbane case studies," in Proceedings of the 2005 AITPM National Conference, J. Douglas, Ed., pp. 229-244.

[5] T. F. Golob, W. W. Recker, and D. W. Levine, "Safety of highoccupancy vehicle lanes without physical separation," Journal of Transportation Engineering, vol. 115, no. 6, pp. 591-607, 1989.

[6] K. Jang, K. Chung, D. R. Ragland, and C.-Y. Chan, "Safety performance of high-occupancy-vehicle facilities," Transportation Research Record, no. 2099, pp. 132-140, 2009.

[7] X. Qi, G. Wu, K. Boriboonsomsin, and M. J. Barth, "Empirical study of lane-changing characteristics on high-occupancyvehicle facilities with different types of access control based on aerial survey data," Journal of Transportation Engineering, vol. 142, no. 1, Article ID 04015034, 2016.

[8] H. Tao, M. G. Foomani, and C. Alecsandru, "A two-step microscopic traffic safety evaluation model of reserved lanes facilities: an arterial case study," in Transportation Research Board 94th Annual Meeting No. 15-3635, 2015.

[9] V. Thamizh Arasan and P. Vedagiri, "Microsimulation study of the effect of exclusive bus lanes on heterogeneous traffic flow," Journal of Urban Planning and Development, vol. 136, no. 1, Article ID 009001QUP, pp. 50-58, 2010.

[10] B. N. Persaud, R. A. Retting, P. E. Garder, and D. Lord, Observational BeforeAfter Study of the Safety Effect of US, Transportation Research Board, National Research Council, 2001.

[11] D. Gettman and L. Head, "Surrogate safety measures from traffic simulation models," Transportation Research Record, no. 1840, pp. 104-115, 2003.

[12] S. Srinivasan, P. Haas, P. Alluri, A. Gan, and J. Bonneson, "Crash prediction method for freeway segments 2 with high occupancy vehicle (HOV) lanes 3," in Transportation Research Board 95th Annual Meeting (No. 16-6333), 2016.

[13] R. Elvik, "The predictive validity of empirical Bayes estimates of road safety," Accident Analysis \& Prevention, vol. 40, no. 6, pp. 1964-1969, 2008.

[14] D. Gettman, L. Pu, T. Sayed, and S. G. Shelby, Surrogate Safety Assessment Model and Validation: Final Report, 2008, No. FHWA-HRT-08-051.

[15] A. Laureshyn, Å. Svensson, and C. Hydén, "Evaluation of traffic safety, based on micro-level behavioural data: theoretical 
framework and first implementation," Accident Analysis \& Prevention, vol. 42, no. 6, pp. 1637-1646, 2010.

[16] W. Young, A. Sobhani, M. G. Lenné, and M. Sarvi, "Simulation of safety: A review of the state of the art in road safety simulation modelling," Accident Analysis \& Prevention, vol. 66, pp. 89-103, 2014.

[17] J. Archer, "Developing the potential of micro-simulation modelling for traffic safety assessment," in Proceedings of the 13th ICTCT Workshop, vol. 44, 2000.

[18] J. Archer, Methods for the Assessment and Prediction of Traffic Safety at Urban Intersections and Their Application in MicroSimulation Modelling, Royal Institute of Technology, 2004.

[19] A. Sobhani, W. Young, and M. Sarvi, "A simulation based approach to assess the safety performance of road locations," Transportation Research Part C: Emerging Technologies, vol. 32, pp. 144-158, 2013.

[20] C. Hydén, The Development of a Method for Traffic Safety Evaluation: The Swedish Traffic Conflicts Technique, Bulletin Lund Institute of Technology, 1987.

[21] S. J. Older and B. R. Spicer, "Traffic conflicts-a development in accident research," Human Factors: The Journal of Human Factors and Ergonomics Society, vol. 18, no. 4, pp. 335-350, 1976.

[22] F. H. Amundsen and C. Hyden, "Proceedings of first workshop on traffic conflicts," in Proceedings of Workshop on Traffic Conflicts, TTI, Oslo, Norway, 1977.

[23] S. R. Perkins and J. L. Harris, "Traffic conflict characteristicsaccident potential at intersections," in Proceedings of the Traffic Safety and presented at the 47th Annual Meeting, pp. 35-43, Highway Research Board, 1968.

[24] M. R. Parker and C. V. Zegeer, Traffic Conflict Techniques for Safety and Operations: Engineers Guide, 1989.

[25] E. Hauer and P. Garder, "Research into the validity of the traffic conflicts technique," Accident Analysis \& Prevention, vol. 18, no. 6, pp. 471-481, 1986.

[26] K. El-Basyouny and T. Sayed, "Safety performance functions using traffic conflicts," Safety Science, vol. 51, no. 1, pp. 160-164, 2013.

[27] R. Fan, H. Yu, P. Liu, and W. Wang, "Using VISSIM simulation model and surrogate safety assessment model for estimating field measured traffic conflicts at freeway merge areas," IET Intelligent Transport Systems, vol. 7, no. 1, pp. 68-77, 2013.

[28] F. Huang, P. Liu, H. Yu, and W. Wang, "Identifying if VISSIM simulation model and SSAM provide reasonable estimates for field measured traffic conflicts at signalized intersections," Accident Analysis \& Prevention, vol. 50, pp. 1014-1024, 2013.

[29] M. Essa and T. Sayed, "Simulated traffic conflicts: do they accurately represent field-measured conflicts?" Transportation Research Record, vol. 2514, pp. 48-57, 2015.

[30] PTV VISSIM 6 User Manual, Karlsrule, Germany, 2013.

[31] S. Jackson, L. Miranda-Moreno, P. St-Aubin, and N. Saunier, "Flexible, mobile video camera system and open source video analysis software for road safety and behavioral analysis," Transportation Research Record, no. 2365, pp. 90-98, 2013.

[32] S. Mirjalili and A. Lewis, "The whale optimization algorithm," Advances in Engineering Software, vol. 95, pp. 51-67, 2016.

[33] G. R. Brown, "Traffic conflicts for road user safety studies," Canadian Journal of Civil Engineering, vol. 21, no. 1, pp. 1-15, 1994. 


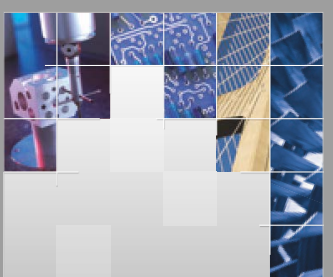

\section{Enfincering}
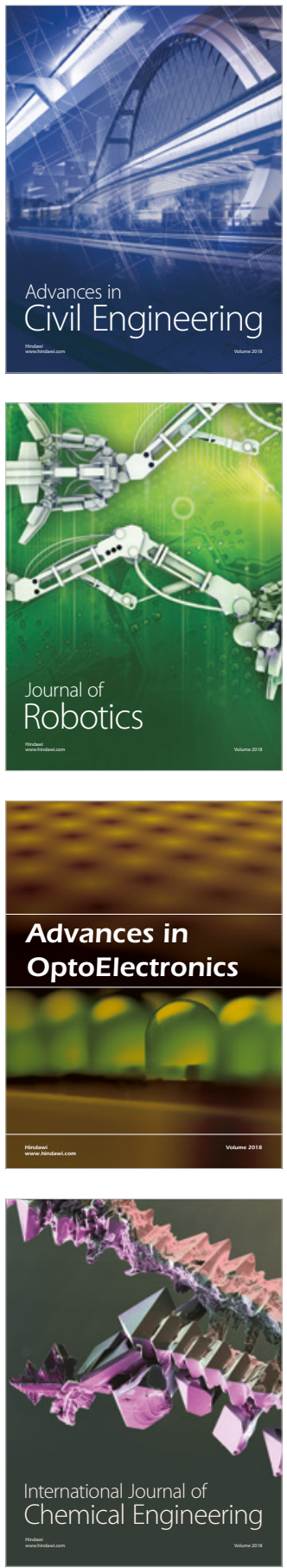

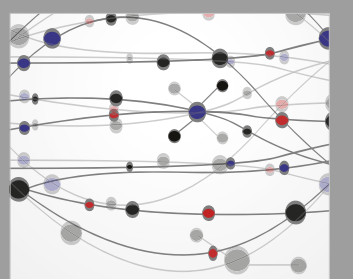

\section{Rotating \\ Machinery}

The Scientific World Journal

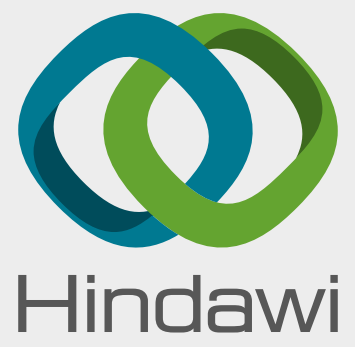

Submit your manuscripts at

www.hindawi.com
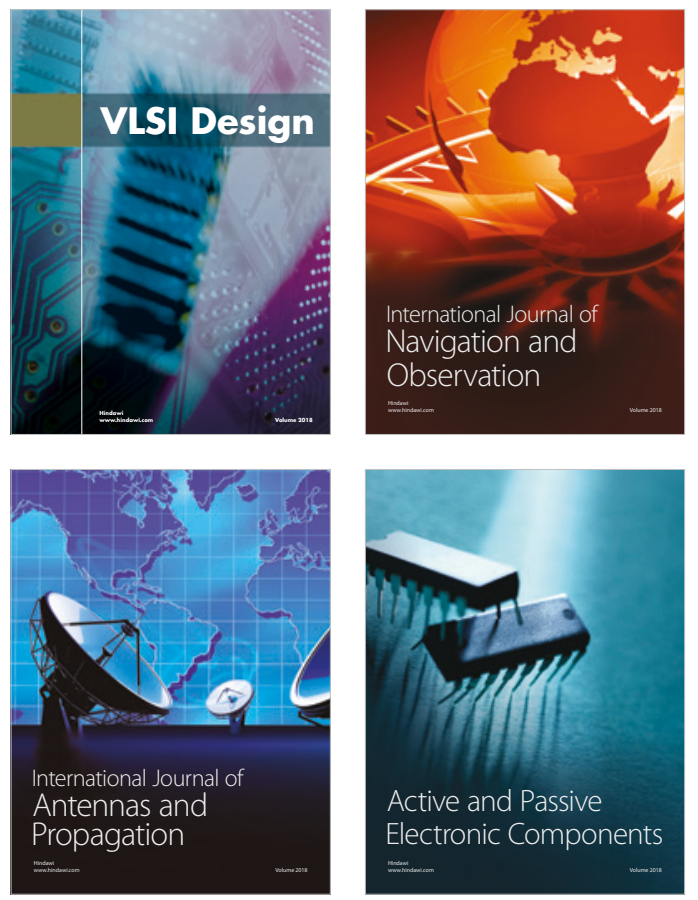
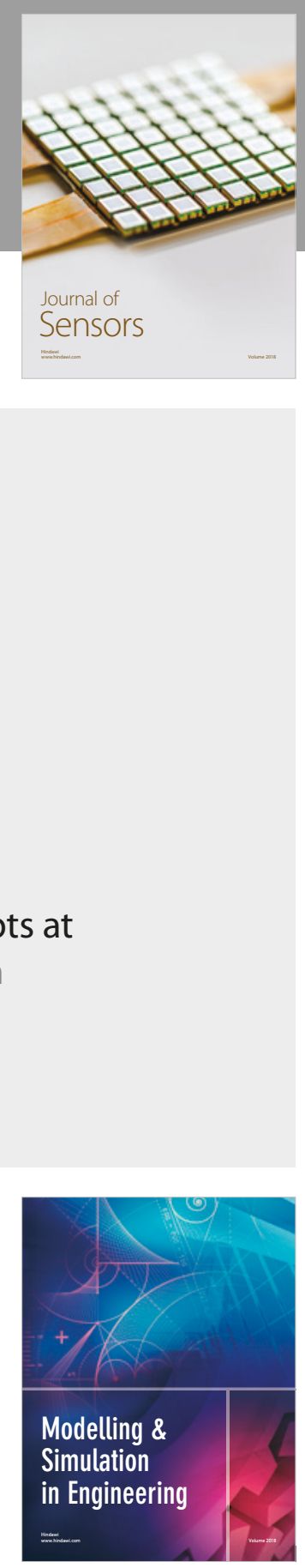

\section{Advances \\ Multimedia}
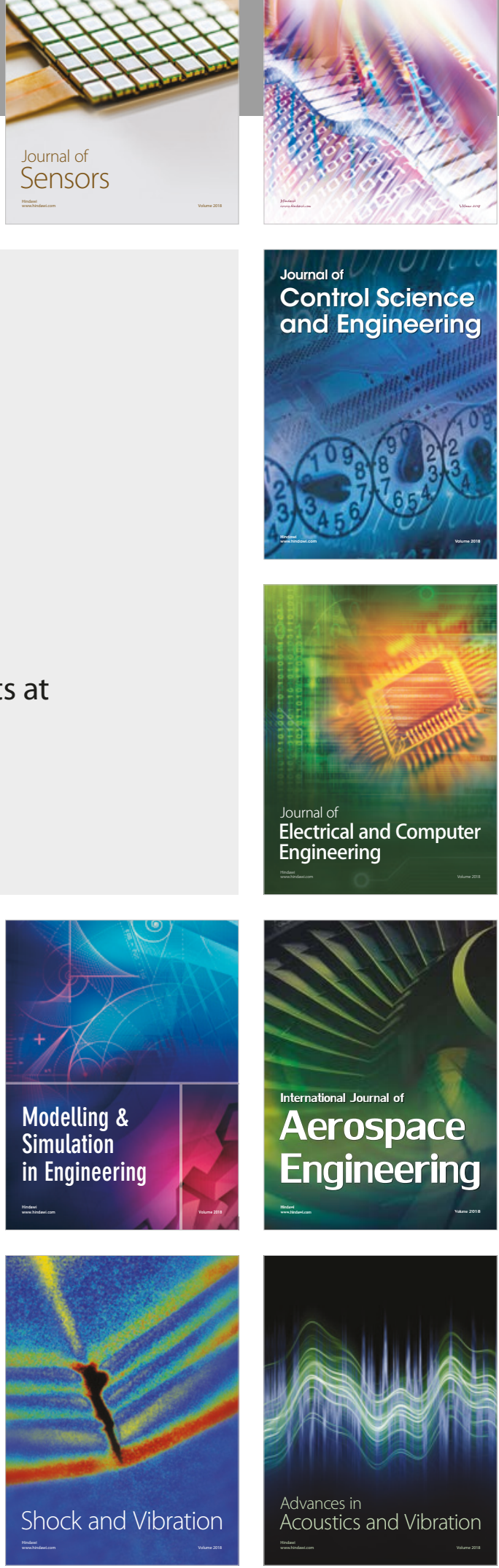